Canad. J. Math. Vol. 54 (5), 2002 pp. 897-915

\title{
The Valuative Theory of Foliations
}

\author{
Pedro Fortuny Ayuso
}

Abstract. This paper gives a characterization of valuations that follow the singular infinitely near points of plane vector fields, using the notion of L'Hôpital valuation, which generalizes a well known classical condition. With that tool, we give a valuative description of vector fields with infinite solutions, singularities with rational quotient of eigenvalues in its linear part, and polynomial vector fields with transcendental solutions, among other results.

\section{Introduction}

The purpose of this paper is to characterize valuations which follow the infinitely near points given by a singularity of a holomorphic vector field on a surface or, what is the same, a germ of a singular first order differential equation in the complex plane. Conceptually, a germ of vector field is nothing but a way of measuring infinitesimal approximations to a local family of curves (the leaves of the associated foliation). Finding a solution through a point is the same as obtaining a subvariety which has infinite contact with the field. Likewise, valuations are the generalization of contact along a local object. Quoting Seidenberg [9], “... derivations are related to contact, and so are valuations, so one may ask for a study connecting derivations and valuations".

To that end, we use the concept of L'Hôpital valuation, defined in Section 2, as a generalization of what Rosenlicht [6], [7] and Singer [10] call differential valuations; a valuation $\nu$ is L'Hôpital for a vector field $\partial$ if it satisfies the following valuative version of classical L'Hôpital's condition:

$$
\nu\left(\frac{a}{b}-\frac{\partial a}{\partial b}\right)>0
$$

whenever $\nu(a) \geq \nu(b)>0$ and $\partial b \neq 0$. The key result of the paper is Theorem 3 of Section 3, which states, essentially, that a L'Hôpital valuation of a vector field follows (after a finite number of blowing-ups) infinitely near singular points of the field. From this it follows that valuations associated to curves which are not leaves of the foliation are not L'Hôpital for the field. Section 3 is devoted to the statement and proof of the main results. We single out two: the equivalence between the existence of an infinite number of invariant analytic curves through the origin (dicriticalness) and L'Hôpital's condition for divisorial valuations (Theorem 7), and the intrinsic

\footnotetext{
Received by the editors June 20, 2001; revised October 30, 2001.

Partially supported by the European Commission: TMR Network "Singularidades de Ecuaciones Diferenciales y Foliaciones", ERBFMRXCT960040. The author wishes to thank Prof. J. M. Aroca for his constant support during the preparation of his Ph.D. thesis.

AMS subject classification: Primary: 12J20, 13F30, 16W60; secondary: 37F75, 34M25.

(c) Canadian Mathematical Society 2002.
} 
characterization of simple singularities with rational quotient of eigenvalues in terms of valuations of rank 1 and rational rank 2 (Theorem 14). In the last two sections, we study the relation of rank 1 valuations with the existence of transcendental solutions (Section 4), and a weak L'Hôpital condition, which allows one to find all the solutions of a vector field, whether singular or not (Section 5).

In the first section we recall, for the sake of both completeness and fixing of notation, the classification of valuations centered in $\mathbb{C}\{x, y\}$ done by Spivakovsky [11] for 2-dimensional regular local rings.

Let us remark also that the present work introduces a new algebraic perspective in the study of singularities of plane and higher dimensional foliations (see [2]), which up to now have been studied mainly with complex analytic tools-either complex analysis or resolution of singularities. From the point of view of differential algebra, the results presented here give an alternative approach to Kolchin-Morrison's theory of continuous derivations [3], [4].

This paper contains the research of the author's Ph.D. thesis concerning complex plane foliations. Results in higher dimension will appear in a forthcoming work.

The author wishes to thank the referee for her/his comments.

\section{Valuations Centered in $\mathbb{C}\{x, y\}$ and Blowing-Ups}

We are mainly interested in valuations centered in a point of the complex plane. Thus, we shall work over the ring $\mathcal{O}=\mathcal{O}_{2}$ of germs of holomorphic functions at a point $P \in \mathbb{C}^{2}$, near which we fix local analytic coordinates $(x, y)$, so that $\mathcal{O} \simeq \mathbb{C}\{x, y\}$. More precisely, if $\mathcal{M}=\mathcal{O}_{(0)}$ denotes the field of meromorphic functions at $P$, we are going to study valuations $\nu$ of $\mathcal{M}$, centered in the local ring $(\mathcal{O}, \mathrm{m})$, that is, such that $\nu(\mathcal{O}) \geq 0$ and $\nu(\mathfrak{m})>0$. In this section we fix some notation and terminology concerning these objects. All the results are well-known since (at least) Zariski [13], although our main reference will be Spivakovsky's work [11], where one can find a general study of valuations centered in noetherian rings of dimension 2, without restrictions on the characteristic. For a complete and up-to-date survey of the general theory of valuations, we refer the reader to [12].

Let $\nu: \mathcal{M}^{\star} \rightarrow \Gamma$ be a valuation of $\mathcal{M}$ centered in $\mathcal{O}$ and consider the blowing-up $\pi: y \rightarrow\left(\mathbb{C}^{2}, 0\right)$ with center $(0,0)$. As $\pi$ is a birational morphism, the field of fractions of $y$ is $\mathcal{M}$, so that it makes sense to speak of $\nu$ as being centered at a point $Q \in \mathcal{Y}$. Call $\tilde{\mathcal{O}}$ to the sheaf of holomorphic functions in $y$. The following result links valuations in $\mathcal{M}$ centered in $\mathcal{O}$ with valuations centered at points of $E:=\pi^{-1}(0,0) \subset y$. The proof is an easy exercise:

Lemma 1 One and only one of the following statements holds:

(1) $\nu$ is the $\mathrm{m}$-adic valuation at $\left(\mathbb{C}^{2}, 0\right)$. That is, if $f \in \mathcal{O}=\mathbb{C}\{x, y\}$, then $\nu(f)=$ $\operatorname{ord}_{(x, y)}(f)$.

(2) There is one and only one closed point $Q \in E$ such that $\nu$ is centered in $Q$. Moreover, $\nu$ can be extended to a valuation of $\tilde{\mathcal{M}}$, the field of fractions of $\tilde{\mathcal{O}}_{Q}$, which is centered in $\tilde{\mathcal{O}}_{Q}$. 
(The extended valuation of 2 will be denoted by $\nu$, for the sake of simplicity). This lemma is the basis of the classification of valuations made by Zariski [13] and Spivakovsky [11], as it allows a grouping in terms of co-final behaviour under blowingups. The first step is to distinguish between divisorial and non-divisorial valuations:

Corollary 2 Let $\nu$ be a valuation of $\mathcal{M}$ centered in $\mathcal{O}$. One and only one of the following statements holds:

(1) There is a finite sequence of blowing-ups

$$
x_{n+1} \stackrel{\pi_{n}}{\longrightarrow} x_{n} \longrightarrow \cdots \stackrel{\pi_{1}}{\longrightarrow} x_{1} \stackrel{\pi_{0}}{\longrightarrow} X_{0}=\left(\mathbb{C}^{2}, 0\right)
$$

(where $n \geq-1$ ) such that if $E_{i+1}$ is the exceptional line of $\pi_{i}$ and $Q_{i+1} \in E_{i+1}$ is the center of the blowing-up $\pi_{i+1}$, then $\nu$ is centered in $Q_{i+1}$ for any $i=-1, \ldots$, $n-1$, and if $(u, v)$ are local coordinates at $Q_{n+1} \in X_{n+1}$, then $\nu$ is the $(u, v)$-adic valuation. In this case, $\nu$ is called the divisorial valuation with strict center $Q_{n+1}$.

(2) There is an infinite sequence of blowing-ups

$\cdots \longrightarrow X_{n+1} \stackrel{\pi_{n}}{\longrightarrow} X_{n} \longrightarrow \stackrel{\pi_{1}}{\longrightarrow} X_{1} \stackrel{\pi_{0}}{\longrightarrow} X_{0}=\left(\mathbb{C}^{2}, 0\right)$

such that if $E_{i+1}$ is the exceptional line of $\pi_{i}$ and $Q_{i+1} \in E_{i+1}$ is the center of the blowing-up $\pi_{i+1}$, then $\nu$ is centered in $Q_{i+1}$ for any $i \geq-1$.

In both cases, the chain of blowing-ups is said to be associated to $\nu$.

In order to classify non-divisorial valuations, we introduce some terminology. Suppose $\nu$ is non-divisorial and let $X_{n+1} \stackrel{\pi}{\longrightarrow} X_{0}=\left(\mathbb{C}^{2}, 0\right)$ be the composition of the first $n$ blowing-ups of the sequence $\left(\pi_{i}\right)_{i \geq 0}$ associated to $\nu$. The fiber $F_{n+1}$ of 0 is the union of $n+1$ irreducible components

$$
F_{n+1}=E_{1} \cup \cdots \cup E_{n+1},
$$

where $E_{i+1}$ is the exceptional line of the blowing-up $\pi_{i}$.

Definition 1 The line $E_{i+1}$ is called the $(i+1)$-st irreducible component of $F_{n+1}$, for $i=0, \ldots, n$.

Definition 2 We say that $Q_{n+1}$ is a corner if it belongs to two different irreducible components of $F_{n+1}$. Otherwise, we say that it is a regular point of $F_{n+1}$.

These notions allow one to group non-divisorial valuations of $\mathcal{M}$ centered in $\mathcal{O}$ into four different families. Recall that any valuation $\nu$ of $\mathcal{M}$ can be naturally extended to one and only one valuation $\hat{\nu}$ of $\hat{\mathcal{M}}=\mathbb{C}((x, y))$.

Definition 3 Fix a valuation $\nu$ centered in $\mathcal{O}$. We say that 
(1) $\nu$ is associated to a curve, or is of contact with a curve if there is an $n_{0} \in \mathbb{N}$ such that for any $m \geq n_{0}$, the point $Q_{m}$ is a regular point of $F_{m}$. In this case, there exists an irreducible principal ideal $(\hat{f}) \in \hat{\mathcal{O}}=\mathbb{C}[[x, y]]$ such that the valuation $\hat{\nu}$ has value group $\mathbb{Z}^{2}$, with the lexicographic order, and is given by the condition

$$
\nu(\hat{g})=(i, j) \Longleftrightarrow \hat{g} \in(\hat{f})^{i}-(\hat{f})^{i+1} \text { and } \quad \#\left(\frac{\hat{g}}{\hat{f}^{i}}, \hat{f}\right)=j,
$$

where \# indicates the ordinary intersection multiplicity. There are two subcases: either $(\hat{f}) \cap \mathcal{O}=(0)$, in which case $\nu$ is said to be associated to a formal curve, or $(\hat{f}) \cap \mathcal{O} \neq(0)=(f)$, where $f$ is irreducible. In this case, $\nu$ is associated to a convergent curve. If no adjective is present, a valuation associated to a curve is assumed to be associated to a convergent one. Notice that in the non-convergent case, if $\left(t^{\alpha}, \varphi(t)\right)$ is a Puiseux parametrization of $(\hat{f}=0)$, then the value of any $g \in \mathcal{O}$, is exactly

$$
\nu(g)=\operatorname{ord}_{t}\left(g\left(t^{\alpha}, \varphi(t)\right)\right) .
$$

(2) $\nu$ is of contact with a divisor if there is $n_{0} \in \mathbb{N}$ such that $m \geq n_{0}$ implies $\pi_{m+1}$ is centered in the intersection of $E_{m}$ and $E_{n_{0}}$. In fact, these valuations can be understood as associated to the curve $E_{n_{0}}$ at the point $E_{m} \cap E_{n_{0}}$ in the birational model $X_{m}$ of $\mathcal{M}$. The points $Q_{m}=E_{n_{0}} \cap E_{m}$ will be called the fixed corners of $\nu$.

(3) $\nu$ has an irrational Puiseux exponent if for $m \gg 0$, the point $Q_{m}$ is a corner of $F_{m}$, but these corners do not share a common divisor (which is case 2): $\nu$ "jumps" from divisor to divisor, but is always centered in corners. For $m \gg 0$, there is a local system of coordinates $(u, v)$ at $Q_{m}$ and $\lambda \in \mathbb{R}_{>0}-\left(\mathbb{O}_{>0}\right.$ such that if $f(u, v)=\sum a_{i j} u^{i} v^{j} \in \hat{\mathcal{O}}_{Q_{m}} \cap \hat{\mathcal{M}}$, then

$$
\nu(f)=\nu\left(\sum_{i, j>0} a_{i j} u^{i} v^{j}\right)=\min _{a_{i j} \neq 0} \lambda i+j .
$$

This $\lambda$ depends on $m$, and can be constructed by a Bezout-type algorithm (see [11]).

(3) $\nu$ has an infinite number of Puiseux exponents if in the sequence of centers of $\nu$ there is an infinite number of both corners and regular points. In [11] one can see the proof of the following fact: there is a minimal generating sequence $\left(Q_{i}\right)_{i \in \mathbb{N}_{0}}$ (see [11]) for $\nu$, such that each $Q_{i}$ defines an analytically irreducible curve $C_{i}$ with $i-1$ Puiseux exponents, and such that, for $0<i<j$, the curve $C_{i}$ has maximal contact with $C_{j}$, which explains the expression "infinite Puiseux exponents".

Valuations of contact with a divisor (type 2) are to be considered a subclass of those of contact with a curve, for up to birational morphisms, there is no difference between them. Thus, we shall only mention them explicitly in Corollary 11. For the reader's convenience, we recall the following

Definition 4 The rank of a valuation $\nu$, is the dimension of the valuation ring $\mathcal{O}_{\nu}$ : $r k(\nu)=\operatorname{dim} \mathcal{O}_{\nu}$. The rational rank is the dimension of the $(0)$-vector space spanned 
by the value group $\Gamma_{\nu}$ :

$$
\text { rat.rk }(\nu)=\operatorname{dim}_{\mathbb{Q}}\left(\Gamma_{\nu} \otimes_{\mathbb{Z}}(\mathbb{Q})\right. \text {. }
$$

\section{L'Hôpital Valuations}

Let $\mathcal{D}_{\mathcal{O}}=\operatorname{Der}_{\mathbb{C}}^{\mathfrak{m}}(\mathcal{O})$ denote the $\mathcal{O}$-module of inner derivations of $\mathbb{C}\{x, y\}=\mathcal{O}$ trivial over $\mathbb{C}$ and continuous for the $\mathrm{m}$-adic topology. In fact, $\mathcal{D}$ is the $\mathcal{O}$-module of germs of analytic vector fields at $\left(\mathbb{C}^{2}, 0\right)$, which is free of rank 2 . We shall denote by $\Omega_{\mathcal{O}}$ the module of germs of holomorphic 1 -forms over $\left(\mathbb{C}^{2}, 0\right)$, and by $\mathcal{D}_{\mathcal{M}}$ and $\Omega_{\mathcal{M}}$ the $\mathcal{M}$ vector spaces of meromorphic derivations and forms, respectively. For reasons that will become apparent later, we are interested in the projectivization of these vector spaces, namely $\mathcal{D}=\mathcal{D}_{\mathcal{M}}-\{0\} / \mathcal{M}^{\star}$ and $\boldsymbol{\Omega}=\Omega_{\mathcal{M}}-\{0\} / \mathcal{M}^{\star}$, which can be regarded (via the usual pairing) as the same set, called the set of germs of foliations in $\left(\mathbb{C}^{2}, 0\right)$. A (germ of) holomorphic foliation will be an element $\boldsymbol{\partial} \in \mathcal{D}$ (or equivalently, its dual $\boldsymbol{\omega} \in \boldsymbol{\Omega})$.

Let $\nu$ be a valuation of $\mathcal{M}$ and $\boldsymbol{\partial} \in \mathcal{D}$ a foliation. Recall that one defines $\nu(0)=$ $\infty$, where $\infty$ is adjoined to $\Gamma$, with the usual order and addition rules. The relation between foliations and valuations is given by the following definition, which is a generalization of what Rosenlicht calls, in a more restrictive context, differential valuations [5]:

Definition 5 We say that $\nu$ is a L'Hôpital valuation for $\boldsymbol{\partial}$ if for any representative $\partial$ of $\boldsymbol{\partial}$, the following four equivalent conditions hold:

(1) For any $a, b \in \mathcal{M}^{\star}$ with $\nu(a) \geq \nu(b)>0$ and $\partial b \neq 0$,

$$
\nu\left(\frac{a}{b}-\frac{\partial a}{\partial b}\right)>0
$$

(2) For any $a, b \in \mathcal{M}^{\star}$ with $0>\nu(a) \geq \nu(b)$ and $\partial b \neq 0$,

$$
\nu\left(\frac{a}{b}-\frac{\partial a}{\partial b}\right)>0
$$

(3) For any $a, b \in \mathcal{M}^{\star}$ with $\nu(a) \geq 0, \nu(b)>0$ and $\partial b \neq 0$,

$$
\nu\left(\frac{\partial a b}{\partial b}\right)>0 .
$$

(4) For any $a, b \in \mathcal{M}^{\star}$ with $\nu(a) \geq 0, \nu(b)<0$ and $\partial b \neq 0$,

$$
\nu\left(\frac{\partial a b}{\partial b}\right)>0 .
$$


Remark Notice that the definition is independent of the representative of $\boldsymbol{\partial}$, as the derivation appears both in the numerator and the denominator of the left-hand member of each equation.

The above notion is the natural translation of classical L'Hôpital's rule to germs of holomorphic vector fields in the plane: the main difference lying, grosso modo, in the fact that in the one-variable case, there are only one foliation and one valuation (compatible with the local structure of $\mathbb{C}\{t\}$ ), whereas in higher dimensions one needs to fix the foliation and impose conditions on the valuation to relate both concepts.

Example (See [2] and [5]) Consider the differential equation

$$
\frac{d y}{d x}=-\frac{y}{x^{2}}
$$

having the fundamental solution $y=e^{1 / x}$, which is holomorphic in a punctured neighbourhood of $0 \in \mathbb{C}$. Let $\mathcal{K}=\mathbb{C}\left(z, e^{1 / z}\right)$ be the field of rational functions in $z, e^{1 / z}$. Fix two analytic paths $\gamma_{0}, \gamma_{1}$, with $\gamma_{0} \subset\{\operatorname{Re}(z)>0\}, \gamma_{1} \subset\{\operatorname{Re}(z)<0\}$ and $\gamma_{0}(1)=\gamma_{1}(1)=0$. Define $\mathcal{O}^{i}=\left\{f \in \mathcal{K}: \lim _{t \rightarrow 1}\left|f\left(\gamma_{i}(t)\right)\right|<\infty\right\}$, for $i=0,1$. Both $\mathcal{O}^{i}$ are valuation rings. Call $\nu_{i}$ to the valuation associated to $\mathcal{O}^{i}$, and let $\partial \in \operatorname{Der}_{\mathbb{C}} \mathcal{K}$ be the derivation of $\mathcal{K}$ induced by the differential equation: $\partial z=1$, $\partial e^{1 / z}=-1 / z^{2} e^{1 / z}$. It is easy to check (see [5]) that each $\nu_{i}$ is a L'Hôpital valuation for $\partial$. We remark that these rings depend only on "the side of $\mathbb{C}$ where $\gamma$ is in", that is, paths included in $\operatorname{Re}(z)>0$ give the same $\operatorname{ring} \mathcal{O}^{0}$ and paths in $\operatorname{Re}(z)<0$ give $\mathcal{O}^{1}$. This leads us to think that L'Hôpital valuations are also a means to find Stokes lines for ordinary differential equations, although this remains, up to date, a conjecture.

\section{Valuations and Vector Fields in Dimension 2}

We have presented the necessary background to study the links between germs of foliations in $\left(\mathbb{C}^{2}, 0\right)$ and valuations centered in $\mathcal{O}$. As we have remarked, we are going to use both the languages of 1 -forms and of vector fields (distributions), as they are equivalent in this dimension.

Take a holomorphic 1-form $\omega$ and fix a regular system of parameters $(x, y)$ in $\left(\mathbb{C}^{2}, 0\right)$. As $\mathcal{O} \simeq \mathbb{C}\{x, y\}, \omega$ can be written

$$
\omega=a d x+b d y
$$

with $a, b$ holomorphic functions in $\left(\mathbb{C}^{2}, 0\right)$. Given such a form, we have its line of dual vector fields $[\partial]$ generated by

$$
\left\{\begin{array}{l}
\partial x=-b \\
\partial y=a
\end{array}\right.
$$

We might get any non-zero vector field in $[\partial]$, by the remark following Definition 5 . From now on, $\omega$ and the above $\partial$ are fixed. We say that $(0,0)$ is a singular point for a 
foliation $\boldsymbol{\omega} \in \boldsymbol{\Omega}$ if there is a coordinate system $(x, y)$ such that for any representative $\omega=a(x, y) d x+b(x . y) d y$ of $\boldsymbol{\omega}, a(0,0)=b(0,0)=0$.

By a separatrix of $\omega$ we shall mean a formal irreducible curve tangent to $\omega$. That is, a separatrix is a principal irreducible ideal $(\hat{f}) \subset \mathbb{C}[[x, y]]$ such that $d \hat{f} \wedge \omega=\hat{f} \eta$, with $\eta$ a formal 2 -form. From the point of view of vector fields, a separatrix can be defined as a local non-zero $\mathbb{C}$-morphism

$$
\varphi: \mathbb{C}[[x, y]] \rightarrow \mathbb{C}[[t]]
$$

such that

$$
\left\{\begin{array}{l}
\partial \varphi(x) / \partial t=-b(\varphi(x), \varphi(y)) \\
\partial \varphi(y) / \partial t=a(\varphi(x), \varphi(y))
\end{array}\right.
$$

\subsection{The Key Results}

Fix a valuation $\nu$ of $\mathcal{M}=\mathbb{C}\{\{x, y\}\}, \nu$ centered in $\mathcal{O}$. Let $y \stackrel{\pi}{\rightarrow} X$ be the blowing-up of $\mathcal{X}=\left(\mathbb{C}^{2}, 0\right)$ at 0 and call $E=\pi^{-1}(0)$, the exceptional divisor. Suppose there is a point $Q \in E$ such that $\nu$ is centered in $Q$ (that is, $\nu$ is not the m-adic valuation). Let $\mathcal{O}_{Q}$ be the local ring at $Q$ and $\tilde{\omega}$ (respectively $\tilde{\partial}$ ) be the strict transform of $\omega$ at $Q$ (resp. the strict transform of $\partial$ ). The following result is the cornerstone of this work:

Theorem 3 If $\nu$ is a L'Hôpital valuation for $\partial$ and $E$ is invariant for $\tilde{\omega}$ (that is to say, $(0,0)$ is not dicritical for $\omega)$, then $Q$ is a singular point for $\tilde{\partial}$.

Proof Suppose, in order to get a contradiction, that $Q$ is not singular for $\tilde{\partial}$. After a linear change of coordinates at $\left(\mathbb{C}^{2}, 0\right)$, we may assume that $Q$ is the origin of the following chart of $\pi$ :

$$
\pi:\left\{\begin{array}{l}
x=x_{2} \\
y=x_{2} y_{2}
\end{array}\right.
$$

Let $\omega$ be the dual form of $\partial$ and $\tilde{\omega}$ its strict transform. As $(0,0)$ is non-dicritical for $\omega$ and $Q$ is not singular for $\tilde{\omega}$, the starting form can be written as

$$
\omega=x_{2}^{\alpha} u\left(d x_{2}+x_{2} \psi d y_{2}\right)
$$

where $\psi \in \mathcal{O}_{Q}$ (in fact, it is in $\mathcal{O} \subset \mathcal{O}_{Q}$ ) and $u$ is an element of $\mathcal{O}$ not zero at $\left(x_{2}=0, y_{2}=0\right)$. The factor $x_{2}$ appears because $E$ is invariant ( $\omega$ is non-dicritical at $(0,0))$. As $\nu\left(x_{2}\right), \nu\left(y_{2}\right)>0$, it is clear that $\nu(y)>\nu(x)>0$. The vector field $\tilde{\partial}$ is then

$$
\left\{\begin{array}{l}
\tilde{\partial} x_{2}=-x_{2}^{\alpha+1} u \psi \\
\tilde{\partial} y_{2}=x_{2}^{\alpha} u
\end{array}\right.
$$

whence

$$
\nu\left(\frac{y}{x}-\frac{\partial y}{\partial x}\right)=\nu\left(y_{2}-\frac{x_{2} \tilde{\partial} y_{2}+y_{2} \tilde{\partial} x_{2}}{\tilde{\partial} x_{2}}\right)=\nu\left(-\frac{x_{2}^{\alpha+1} u}{-x_{2}^{\alpha+1} u \psi}\right)
$$


but $\nu(u)=0, \nu(1)=0, \nu(\psi) \geq 0$ and $\nu$ cannot be a L'Hôpital valuation for $\partial$.

From Theorem 3 and Seidenberg's reduction of singularities for plane vector fields [8], we infer immediately the following:

Corollary 4 Valuations with an infinite number of Puiseux exponents are never L'Hôpital valuations for any holomorphic vector field $\partial$.

Proof After a finite number of blowing-ups, we may assume that $\partial$ is non-dicritical. Suppose, in order to get a contradiction, that $\nu$ is a L'Hôpital valuation following both regular points and corners of the exceptional divisor in the long run. Seidenberg's result implies [8] that from some $Q_{0}$ onwards, all these points are simple for the strict transform of $\partial$. Assume, without loss of generality, that $Q_{0}$ is corner and both irreducible components $E$ and $E^{\prime}$ of the divisor passing through it are separatrices. From this, we deduce that the only infinitely near singular points of $\partial$ at $Q_{0}$ after the blowing-up $\pi$ with center $Q_{0}$ are the two corners of $\pi^{-1}\left(Q_{0}\right)$ corresponding to $E$ and $E^{\prime}$. Theorem 3 implies that, from $Q_{0}$ onwards, all the infinitely near points of $\nu$ are corners, whence $\nu$ cannot have an infinite number of Puiseux exponents.

Lemma 5 Let $\partial$ be a holomorphic vector field and $\hat{f} \in \mathbb{C}[[x, y]]$ a strictly formal (which means $(\hat{f}) \cap \mathcal{O}=(0))$ separatrix of $\partial$ not tangent to $(x=0)$. Write $\partial=$ $h \partial / \partial x+g \partial / \partial y$ with $(g, h)=1$ and take $u=a / b \in \mathcal{M}$. Suppose $\left(t^{\alpha}, \varphi(t)\right)$ is a Puiseux parametrization of $\hat{f}=0$, with $\operatorname{ord}_{t} \varphi(t)>\alpha$. Then

$$
\partial u\left(t^{\alpha}, \varphi(t)\right)=\frac{1}{\alpha t^{\alpha-1}} h(t) \frac{d}{d t}\left(u\left(t^{\alpha}, \varphi(t)\right)\right) .
$$

Proof We have, by the chain-rule

$$
\partial u=u_{x} \partial x+u_{y} \partial y=u_{x} h+u_{y} g=h\left(u_{x}+\frac{g}{h} u_{y}\right) .
$$

As $\hat{f}=0$ is strictly formal, $h\left(t^{\alpha}, \varphi(t)\right) \neq 0$ and, from the definition of separatrix, we get:

$$
\frac{g}{h}\left(t^{\alpha}, \varphi(t)\right)=\frac{\varphi^{\prime}(t)}{\alpha t^{\alpha-1}},
$$

so that, substituting this expression in the previous one:

$$
\partial(u)\left(t^{\alpha}, \varphi(t)\right)=\frac{1}{\alpha t^{\alpha-1}} h(t) \frac{d}{d t}\left(u\left(t^{\alpha}, \varphi(t)\right)\right),
$$

as desired. 


\subsection{The Dicritical Case}

The link between valuations and dicritical centers is founded on the following:

Lemma 6 Let $\partial$ be a holomorphic vector field in $\left(\mathbb{C}^{2}, 0\right)$ and let $\nu$ be the $\mathrm{m}$-adic valuation centered at $\mathcal{O}$ (that is, $\nu(a)=n \in \mathbb{N}$ if and only if $a \in \mathrm{m}^{n}-\mathrm{m}^{n+1}$ ). Put $r=\min \{\nu(\partial x), \nu(\partial y)\}$. Then

(1) $\nu(a \partial b-b \partial a) \geq \nu(a)+\nu(b)+r-1$, for any $a, b \in \mathcal{O}$.

(2) $\partial$ is dicritical in $(0,0)$ if and only if there are $a, b \in \mathcal{O}$ for which the strict inequality holds. Moreover, in this case, $\nu(a)=\nu(b)$.

Proof Take $a, b \in \mathcal{O} \subset \mathbb{C}[[x, y]]$ with $\nu(a)=m, \nu(b)=n$. Call $\theta=\partial x$ and $\eta=\partial y$ and write $a, b, \theta$ and $\eta$ as power series expansions:

$$
\left\{\begin{array} { l } 
{ a = a _ { m } + a _ { m + 1 } + \cdots } \\
{ b = b _ { n } + b _ { n + 1 } + \cdots }
\end{array} \quad \left\{\begin{array}{l}
\theta=\theta_{l}+\theta_{l+1}+\cdots \\
\eta=\eta_{k}+\eta_{k+1}+\cdots
\end{array}\right.\right.
$$

Applying $\partial$ to $a$ and $b$, we get

$$
\left\{\begin{array}{l}
\partial a=a_{m x} \theta_{l}+a_{m y} \eta_{k}+\cdots \\
\partial b=b_{n x} \theta_{l}+b_{m y} \eta_{k}+\cdots
\end{array}\right.
$$

where subindices $x$ and $y$ mean "ordinary partial derivation". From this last expression, it follows that

$$
\nu(a \partial b-b \partial a) \geq m+n-1+r,
$$

which is the general inequality.

To prove the second assertion, notice that when applying $\partial$ to a monomial $a_{i j} x^{i} y^{j}$, one gets

$$
\partial\left(a_{i j} x^{i} y^{j}\right)=i a_{i j} x^{i-1} y^{j} \theta+j a_{i j} x^{i} y^{j-1} \eta
$$

and, looking at the terms of least degree in $\theta$ and $\eta$, we have

$$
\partial\left(a_{i j} x^{i} y^{j}\right)=i a_{i j} x^{i-1} y^{j} \theta_{r}+j a_{i j} x^{i} y^{j-1} \eta_{r}+\text { h.o.t. }
$$

hence

$$
\begin{aligned}
& \partial\left(a_{i j} x^{i} y^{j}\right) b_{p q} x^{p} y^{q}-\partial\left(b_{p q} x^{p} y^{q}\right) a_{i j} x^{i} y^{j} \\
& =\left(i a_{i j} x^{i-1} y^{j} \theta_{r}+j a_{i j} x^{i} y^{j-1} \eta_{r}\right) b_{p q} x^{p} y^{q} \\
& \quad-\left(p b_{p q} x^{p-1} y^{q} \theta_{r}+q b_{p q} x^{p} y^{q-1} \eta_{r}\right) a_{i j} x^{i} y^{j}+\text { h.o.t } \\
& =\left((i-p) a_{i j} b_{p q} \theta_{r}\right) x^{i+p-1} y^{j+q}+\left((j-q) a_{i j} b_{p q} \eta_{r}\right) x^{i+p} y^{j+q-1}+\text { h.o.t. }
\end{aligned}
$$

which is zero if and only if $i+j=p+q$ (that is, $\nu(a)=\nu(b))$ and $x \theta_{r}+y \eta_{r}=0$ (i.e., $\partial$ is dicritical at $(0,0))$, and the conclusion follows.

Lemma 6 allows us to classify dicritical vector fields in terms of L'Hôpital valuations: 
Theorem 7 Let $\nu$ be a divisorial valuation of $\mathcal{M}$, centered at $\mathcal{O}$. Let $Q$ be the strict center (see Corollary 2) of $\nu$ and $\partial$ a foliation by curves on $\left(\mathbb{C}^{2}, 0\right)$. Then

$$
\nu \text { is a L'Hôpital valuation for } \partial \Longleftrightarrow \partial \text { is dicritical at } Q \text {. }
$$

Proof Fix a local system of parameters $(u, v)$ at $Q, u, v \in \mathcal{O}_{Q}$. There are $\sigma, \theta, \eta \in \mathcal{O}_{Q}$ with coefficients in $\mathcal{O}_{Q}$ such that $\tilde{\partial}$ can be written as

$$
\tilde{\partial}=\frac{\theta(u, v)}{\sigma(u, v)} \frac{\partial}{\partial u}+\frac{\eta(u, v)}{\sigma(u, v)} \frac{\partial}{\partial v} .
$$

Moreover, in those coordinates, $\nu=\operatorname{ord}_{(u, v)}$ is "the order in $(u, v)$ ". We can forget the denominators and suppose $\theta$ and $\eta$ are relatively prime in $\mathcal{O}_{Q}$. Let $m$ be the smallest among $\operatorname{ord}_{(u, v)}(\theta), \operatorname{ord}_{(u, v)}(\eta)$. For any two meromorphic functions $a=$ $\bar{a}(u, v) / \Delta(u, v), b=\bar{b}(u, v) / \Delta(u, v)$ with $\bar{a}, \bar{b}, \Delta \in \mathcal{O}_{Q}$, one has

$$
\frac{a}{b}-\frac{\partial a}{\partial b}=\frac{\Delta(\bar{a} \partial \bar{b}-\bar{b} \partial \bar{a})}{\bar{b}(\Delta \partial \bar{b}-\bar{b} \partial \Delta)}
$$

Let $d=\operatorname{ord}(\Delta), s=\operatorname{ord}(\bar{b}), t=\operatorname{ord}(\bar{a})$ and suppose, in order to study L'Hôpital's condition, that $\nu(a) \geq \nu(b)>0$, which means that $0 \leq d<s \leq t$. Lemma 6 asserts that

$$
\nu(\Delta(\bar{a} \partial \bar{b}-\partial \bar{a} \bar{b})) \geq \bar{b}(\Delta \partial \bar{b}-\bar{b} \partial \Delta)=2 s+d+m-1 .
$$

The last equality holds because ord $(\bar{b})>\operatorname{ord}(\Delta)$. L'Hôpital's condition means that the order of the numerator is greater that the order of the denominator. This follows easily if $t>s$. When $t=s$, the condition follows if and only if ( $c f$. Lemma 6) $\partial$ is dicritical at $Q$, which finishes the proof.

\subsection{Separatrices}

The first result relating solutions of differential equations with L'Hôpital valuations deals with strictly formal separatrices:

Theorem 8 Let $\partial$ be a foliation by curves in $\left(\mathbb{C}^{2}, 0\right)$ and $\nu$ the valuation associated to a formal curve (see Definition 3) $(\hat{f}=0)$. Then

$$
\hat{f}=0 \text { is a separatrix of } \partial \Longleftrightarrow \nu \text { is a L'Hôpital valuation for } \partial \text {. }
$$

Proof $\Leftarrow)$ This is an easy consequence of Theorem 3 and the fact that a (formal) curve following singular points of the strict transforms of $\partial$ by blowing-ups is a separatrix.

$\Rightarrow \nu$ has rank 1 and, in fact, is the intersection multiplicity with $\hat{f}$, which means that if $\left(x=t^{\alpha}, y=\varphi(t)\right)$ is a Puiseux parametrization of $\hat{f}=0$ with $\operatorname{ord}_{t}(\varphi(t))>$ $\alpha$ then, for $a=a(x, y) \in \mathcal{O}$, we have $\nu(a)=\operatorname{ord}_{t}\left(a\left(t^{\alpha}, \varphi(t)\right)\right)$. Let us prove 
that, in fact, it is a L'Hôpital valuation with respect to $\partial$. Write $\partial=h \frac{\partial}{\partial x}+g \frac{\partial}{\partial y}$ with $h, g \in \mathcal{O}$ and relatively prime in $\mathcal{O}$. By Lemma 5 , if $p, q \in \mathcal{M}$, then

$$
\nu\left(\frac{p}{q}-\frac{\partial p}{\partial q}\right)=\operatorname{ord}_{t}\left(\frac{p}{q}(t)-\frac{\partial p}{\partial q}(t)\right)=\operatorname{ord}_{t}\left(\frac{p(t)}{q(t)}-\frac{p^{\prime}(t)}{q^{\prime}(t)}\right)>0,
$$

the last equality holding as a consequence of L'Hôpital's rule for one complex variable.

It appears that in order to study valuations associated to convergent separatrices, one needs to distinguish them according to the quotient of eigenvalues of the linear part of the vector field at a point. To be more precise, let $\hat{f}$ be a separatrix, convergent or not, of a holomorphic vector field $\partial$ and let $\rho$ be a resolution of singularities of $\hat{f}$. Put $E=\rho^{-1}(0,0)$. We may assume that the last exceptional line appearing in $E$ is invariant for $\rho^{\star} \partial$, that the strict transform of $\hat{f}$ meets $E$ in a regular point of $E$ and, finally, that $Q$ is a simple point for $\rho^{\star} \partial(c f$. [8]). Let $(u, v)$ be a local regular system of parameters of $\mathcal{O}_{Q}$ such that $u=0$ is the equation of $E$ and $v=0$ is tangent to the strict transform of $\hat{f}$. In these coordinates, the equation of $\rho^{\star} \partial$ has non-zero linear part of the form $\mu u \partial / \partial u-\lambda v \partial / \partial v$.

Definition 6 We say that $\hat{f}$ has

- Generic character if $\lambda \mu \neq 0$ and $\lambda / \mu \notin(0)$.

- Infinite character if $\mu=0$.

- Zero character if $\lambda=0$.

- Rational character if $\lambda \mu \neq 0$ and $\lambda / \mu \in(0)$.

Remark 9 The transformation rule for of the coefficients of the linear part of $\partial$ by blowing-up shows that the character is well defined: it does not depend either on $\rho$ or on the coordinates $(u, v)$.

Theorem 10 If $\nu$ is of contact with the convergent curve defined by the ideal $(f) \subset \mathcal{O}$, then

$$
\nu \text { is a L'Hôpital valuation } \Longleftrightarrow f \text { is a separatrix with }\left\{\begin{array}{c}
\text { generic } \\
\text { or } \\
\text { infinite }
\end{array}\right\} \text { character. }
$$

Proof For $a \in \mathcal{O}, \nu$ is given by

$$
\nu(a)=(i, j) \Longleftrightarrow a \in(f)^{i}-(f)^{i+1}, \quad \#\left(\frac{a}{f^{i}}, f\right)=j,
$$

where $\#(a, b)$ is, as usual, the intersection multiplicity of the curves $(a=0)$ and $(b=0)$.

$\Rightarrow$ ) From Theorem 3, we infer that $(f=0)$ must be a separatrix of $\partial$. As for the character, we divide the proof in two parts: 
a) Assume, first, that $(f=0)$ has rational character. After reducing the singularities of $\partial$ (and hence of $f$ ), we may assume that the eigenvalues of its linear part are 1 and $m / n$, with $m, n \in \mathbb{N}$. We may also assume that the irreducible component $F$ of the exceptional divisor $E$ meeting $(f=0)$ is both invariant for $\partial$ and transversal to $(f=0)$ at a regular point of $E$. Take a local system of parameters at $Q$, say $(u, v)$, such that the strict transform of $f$ follows the same infinitely near points as $(u=0)$ and such that $F$ is given by $(v=0)$. Things being so, the vector field can be written, at $Q$, as follows:

$$
\left\{\begin{array}{l}
\partial u=u\left(1+f_{1}\right) \\
\partial v=-\frac{m}{n} v\left(1+f_{2}\right)
\end{array} \quad \text { with } \nu\left(f_{1}\right), \nu\left(f_{2}\right)>0,\right.
$$

as $f=0$ and $v=0$ are invariant and $\partial$ is simple at $Q$. Take

$$
\left\{\begin{array}{l}
a=u^{m} v^{n+1} \\
b=u^{m} v^{n}
\end{array}\right.
$$

By construction, $\nu(a) \geq \nu(b)>0$. Let us see if they satisfy L'Hôpital's criterion:

$$
\frac{a}{b}-\frac{\partial a}{\partial b}=\frac{v^{2 n+1}\left(m / n+g_{1}\right)}{v^{2 n} g_{2}}=\frac{\alpha}{\beta}
$$

with $\nu\left(g_{1}\right), \nu\left(g_{2}\right)>0$. As $(n+1) m / n \neq 0$, we have $\nu(\alpha)=(0,2 n+1)$ and $\nu(\beta)>(0,2 n)$, so that the pair $a, b$ does not satisfy the criterion and $\nu$ is not L'Hôpital for $\partial$.

b) Suppose that $f=0$ has character zero. The same argument as in a) shows that we can take a local system of coordinates $(u, v)$ at $Q$ with

$$
\left\{\begin{array}{l}
\partial u=u^{q+1}+u f_{1} \\
\partial v=-v\left(1+f_{2}\right)
\end{array} \quad \text { with } \nu\left(f_{1}\right), \nu\left(f_{2}\right)>0, q \geq 1 .\right.
$$

In this situation, take

$$
\left\{\begin{array}{l}
a=u v \\
b=u(u+v) / v
\end{array}\right.
$$

which satisfy $\nu(a) \geq \nu(b)>0$. An easy computation shows that

$$
\frac{a}{b}-\frac{\partial a}{\partial b}=\frac{v^{2}}{u+v}-\frac{-v^{2}-v^{2} f_{2}+v^{2} f_{1}+u^{q} v^{2}}{v f_{1}+u g}
$$

where $g \in \mathcal{O}_{Q}$. As $\nu(u+v)=\nu(v)=(0,1)$ and $\nu\left(f_{1}\right)>0$, the valuation does not satisfy L'Hôpital's criterion, q.e.d.

$\Leftarrow)$ Fix, as before, $Q$ in the exceptional divisor of a reduction of singularities of $\partial$ and $(f=0)$, which is not a corner and take coordinates $(u, v)$ at $Q$ such that $\partial$ is given by

$$
\left\{\begin{array}{l}
\partial u=u\left(1+f_{1}\right) \\
\partial v=-\lambda v-v f_{2}
\end{array} \quad \text { with } \nu\left(f_{1}\right), \nu\left(f_{2}\right)>0 .\right.
$$


Let $a=u^{i} \bar{a}, b=u^{j} \bar{b}$ with $\nu(a) \geq \nu(b)>0$ and $\nu(\bar{a})=(0, k), \nu(b)=(0, l)$. We have

$$
\frac{a}{b}-\frac{\partial a}{\partial b}=\frac{u^{i+j}}{u^{2 j}}\left(\frac{j \bar{a} \bar{b}+j \bar{a} \bar{b} f_{1}+\bar{a} \partial \bar{b}-i \bar{a} \bar{b}-i \bar{a} \bar{b} f_{1}-\partial \bar{a} \bar{b}}{\bar{b}\left((j \bar{b}+\partial \bar{b})+j \bar{b} f_{1}\right)}\right)=u^{i-j} \frac{\alpha}{\beta} \text {. }
$$

Let us make a brief digression in order to calculate $\nu(\beta)$.

Write $\bar{b}=p / q$, with $p, q \in \mathcal{O}_{Q}$ as follows:

$$
\left\{\begin{array}{l}
p=v^{n}+u h_{1}+v^{n+1} h_{2} \\
q=v^{m}+u g_{1}+v^{m+1} g_{2}
\end{array}\right.
$$

where the functions $h_{i}$ and $g_{j}$ are formal power series in $\mathbb{C}[[u, v]]$. Their derivatives are

$$
\left\{\begin{array}{l}
\partial p=-n \lambda v^{n}+u \delta_{1}-\lambda v^{n+1} \delta_{2}+v^{n} f_{2} \delta_{3} \\
\partial q=-m \lambda v^{m}+u \epsilon_{1}-\lambda v^{m+1} \epsilon_{2}+v^{m} f_{2} \epsilon_{3}
\end{array}\right.
$$

where, again, $\delta_{i}, \varepsilon_{j}$ are in $\mathcal{O}_{Q}$. From (3), we have that $\nu(q \partial p-p \partial q) \geq 2 \nu(q)$ and, hence, $\nu(\partial \bar{b}) \geq \nu(\bar{b})$. In fact, equality holds if and only if $n \neq m$ and $\lambda \neq 0$. If $\nu(\partial \bar{b})>\nu(\bar{b})$, then $\nu(\beta)=2 \nu(\bar{b})$. If not, then

$$
j \bar{b}+\partial \bar{b}=j \frac{v^{n}+\cdots}{v^{m}+\cdots}+\frac{\lambda(n-m) v^{n+m}+\cdots}{v^{2 m}+\cdots}=\star,
$$

(dots indicating terms of greater value). But

$$
\star=\frac{j v^{m+n}+\lambda(m-n) v^{n+m}+\cdots}{v^{2 m}+\cdots}
$$

and as, by hypothesis, $\lambda$ is neither zero nor rational, then $j+\lambda(m-n) \neq 0$ when $j \neq 0$, and in this case we get $\nu(j \bar{b}+\partial \bar{b})=\nu(\bar{b})$. If $j=0$, then $n>m$ and hence $\nu(\partial \bar{b})=\nu(\bar{b})$.

Thus, in any case, $\nu(\beta)=2 \nu(\bar{b})$.

Continuing with the proof, if $i>j$, then L'Hôpital's condition is satisfied because of the factor $u^{i-j}$. If $i=j$, then $\nu(\bar{a}) \geq \nu(\bar{b})>0$, and from the expression of $\alpha$ in (2), we infer that

$$
\nu(\alpha) \geq \nu\left(\bar{b}^{2} \partial\left(\begin{array}{c}
\bar{a} \\
\overline{\bar{b}}
\end{array}\right)\right),
$$

from what follows, by a reasoning similar to the previous one, that $\nu(\alpha)>\nu(\beta)$, and we are done.

A straightforward consequence is the following:

Corollary 11 Let $\nu$ be a valuation of contact with a divisor $E_{k}$, and let $\partial$ be a vector field. Let $Q$ be a fixed corner of $\nu$, simple for $\partial$ and such that both components of the exceptional divisor passing through $Q$ are invariant. Then $\nu$ is a L'Hôpital valuation for $\partial$ if and only if one of the following conditions holds:

a) The two irreducible components of the exceptional divisor passing through $Q$ have generic character (understood as separatrices in $\mathcal{O}_{Q}$ ).

b) $E_{k}$ has infinite character (and hence, the other component has character zero). 


\subsection{Valuations with an Irrational Puiseux Exponent}

As in the previous case, we have to distinguish between rational and non-rational quotients of eigenvalues, although in a slightly different way.

Lemma 12 Let $Q$ be a simple singularity of a plane vector field $\partial=b_{1} \partial / \partial x-a_{1} \partial / \partial y$, where $a_{1}, b_{1}$ are of order 1 in $(x, y)$. Assume that the eigenvalues of the linear part of $\partial$ are both non-zero and have non-rational quotient. Then any valuation $\nu$ with an irrational Puiseux exponent following singular infinitely near points of $\partial$ is a L'Hôpital valuation for $\partial$.

Proof Up to a linear change of coordinates, we can write

$$
\left\{\begin{array}{l}
\partial u=\lambda u+\cdots \\
\partial v=v+\cdots
\end{array}\right.
$$

where $(u, v)$ is the new coordinate system. Also we may assume that the associated valuation is $\nu(u)=1, \nu(v)=\kappa \in \mathbb{I}_{+}$, so that if $\alpha=\sum a_{i j} u^{i} v^{j} \in \mathbb{C}[[u, v]]$, then $\nu(\alpha)=\min _{a_{i j} \neq 0}(i+\kappa j)=\operatorname{ord}_{t}\left(\sum a_{i j} t^{i+\kappa j}\right)$. Given $\alpha, \beta \in \mathcal{O}_{Q}$, we have

$$
\partial\left(\frac{\alpha}{\beta}\right)=\frac{\left(\lambda u \alpha_{u}+v \alpha_{v}\right) \beta-\left(\lambda u \beta_{u}+v \beta_{v}\right) \alpha}{\beta^{2}}+\cdots .
$$

The set of monomials in $\left(\lambda u \alpha_{u}+v \alpha_{v}\right)$ whose coefficient is non-zero is the same as that of $\alpha$, as $\lambda$ is irrational. The same happens with $\left(\lambda u \beta_{u}+v \beta_{v}\right)$ and $\beta$. Let $\left(i_{0}, j_{0}\right)$ and $\left(m_{0}, n_{0}\right)$ be such that $\nu(\alpha)=i_{0}+\kappa j_{0}$ and $\nu(\beta)=m_{0}+\kappa n_{0}$. The numerator in (4) has a term of the form:

$$
\left(\lambda i_{0}+j_{0}\right) \alpha_{i_{0} j_{0}} \beta_{m_{0} n_{0}}-\left(\lambda m_{0}+n_{0}\right) \alpha_{i_{0} j_{0}} \beta_{m_{0} n_{0}}=\alpha_{i_{0} j_{0}} \beta_{m_{0} n_{0}}\left(\lambda\left(i_{0}-m_{0}\right)+\left(j_{0}-n_{0}\right)\right),
$$

which is non-zero. If $\left(i_{0}, j_{0}\right) \neq\left(m_{0}, n_{0}\right)$, then the value of the numerator is the sum of the values of $\alpha$ and $\beta$. If, on the contrary, $\left(i_{0}, j_{0}\right)=\left(m_{0}, n_{0}\right)$, then the value of the numerator is strictly greater than the sum of the values of $\alpha$ and $\beta$.

Take now $\alpha / \beta, \gamma / \beta \in \mathcal{M}$. We need to study

$$
\nu\left(\frac{\alpha / \beta}{\gamma / \beta}-\frac{\partial(\alpha / \beta)}{\partial(\gamma / \beta)}\right)=\nu\left(\frac{\beta(\alpha \partial \gamma-\gamma \partial \alpha)}{\gamma(\beta \partial \gamma-\gamma \partial \beta)}\right) .
$$

Suppose $\nu(\alpha) \geq \nu(\gamma)>\nu(\beta) \geq 0$. From the second inequality and the previous considerations, it follows that $\nu(\gamma(\beta \partial \gamma-\gamma \partial \beta))=\nu(\gamma)+\nu(\gamma)+\nu(\beta)$. There are two possibilities: either $\nu(\alpha)>\nu(\beta)$ or $\nu(\alpha)=\nu(\beta)$. In the first case, the value of the numerator in (5) is, at least, $\nu(\alpha)+\nu(\beta)+\nu(\gamma)$, which is greater than the value of the denominator. To see that the same holds for the second case one only needs to use the above argument on the initial components of $\alpha, \beta, \gamma$ and their derivatives.

Remark Obviously, a valuation with an irrational Puiseux exponent which does not follow singular infinitely near points of a vector field is not a L'Hôpital valuation, in view of Seidenberg's reduction of singularities and Theorem 3. 
Lemma 13 Under the same conditions as in Lemma 12, but with rational quotient of eigenvalues (including the case in which one of them is 0 ), no valuation with an irrational Puiseux exponent is L'Hôpital for $\partial$.

Proof By the previous remark, we only need to consider valuations following singular infinitely near points of $\partial$. We divide the proof into two cases: both eigenvalues are non-zero (resonant case) and one of them is zero (saddle-node).

a) Resonant Singularities We can write $\partial$ as

$$
\left\{\begin{array}{l}
\partial u=-m u+\cdots \\
\partial v=n v+\cdots
\end{array}\right.
$$

After a polynomial change of coordinates (see [1]), we may assume that

$$
\partial=-\left(m u+s_{1}(u, v)\right) \frac{\partial}{\partial u}+\left(n v+s_{2}(u, v)\right) \frac{\partial}{\partial v},
$$

with $\nu\left(s_{1}\right), \nu\left(s_{2}\right) \geq \nu(u)+\nu(v)$ and that the valuation is $\nu(u)=1, \nu(v)=\eta \in \mathbb{I}_{>0}$ with $\eta>1{ }^{1}$ Take $\left(i_{0}, j_{0}\right) \neq(0,0)$ such that $\left(-m i_{0}+n j_{0}\right)=0$. For $c u^{i_{0}} v^{j_{0}}$, we have

$$
\begin{aligned}
\nu\left(\partial\left(c u^{i_{0}} v^{j_{0}}\right)\right) & =\nu\left(i_{0} c u^{i_{0}-1} v^{j_{0}} s_{1}(u, v)+j_{0} c u^{i_{0}} v^{j_{0}-1} s_{2}\right) \\
& \geq \min \left\{i_{0}+j_{0} \eta+\eta, i_{0}+j_{0} \eta+1\right\}=i_{0}+j_{0}+1,
\end{aligned}
$$

by definition. Let $r=\left[i_{0}+j_{0} \eta+1\right]$ (integral part). As $j_{0} \neq 0$ and $\eta \in \mathbb{I}$, we have $r<i_{0}+j_{0} \eta+1$. To verify that L'Hôpital's condition is not satisfied, take $\alpha=u^{r}$, $\gamma=u^{i_{0}} v^{j_{0}}$, for which $\nu(\alpha)>\nu(\gamma)>0$, but $\nu(\partial \gamma)>\nu(\partial \alpha)=\nu(\alpha)$, so that

$$
\nu\left(\frac{\alpha}{\beta}-\frac{\partial \alpha}{\partial \beta}\right)=\nu(\alpha)+\nu(\gamma)-\nu(\gamma)-\nu(\partial \gamma)<0
$$

which finishes the proof in this case.

b) Saddle-node The vector field has a representative of the form

$$
\partial=a(u, v) \frac{\partial}{\partial u}+(v+b(u, v)) \frac{\partial}{\partial v}
$$

with $\operatorname{ord}_{(u, v)}(a), \operatorname{ord}_{(u, v)}(b) \geq 2$. The valuation is defined by $\nu(u)=1, \nu(v)=\eta \in$ $\mathbb{I}_{>0}$, but we may not assume $\eta>1$. One can prove [1] that, up to a holomorphic change of coordinates, $\partial$ can be written

$$
\partial=\left(u\left(1+\lambda v^{p}\right)+v R(u, v)\right) \frac{\partial}{\partial u}+v^{p+1} \frac{\partial}{\partial v}
$$

\footnotetext{
${ }^{1}$ Here we are using the fact that $m, n \neq 0$.
} 
for suitable $\lambda \in \mathbb{C}$ and $p \in \mathbb{Z}_{\geq 1}$. Truncating, we deduce that there is $p \geq 1$ and a polynomial change of coordinates such that we may assume $\nu(a(u, v)) \geq 2$, (taking $\left.a(u, v)=u^{p+1}+\cdots\right)$. Thus, for any $m \in \mathbb{N}$, we have

$$
\nu\left(\partial\left(u^{m}\right)\right) \geq m+1
$$

Here we have to distinguish between $\eta<1$ and $\eta>1$. In the first case, let $\alpha=u v$ and $\gamma=u$. A direct computation shows that

$$
\nu\left(\frac{\alpha}{\gamma}-\frac{\partial \alpha}{\partial \gamma}\right)=\nu(\alpha)+\nu(\gamma)-\nu(\gamma)-\nu(\partial \gamma) \leq 0
$$

In the second case, taking $r=[\eta]$ and $\alpha=v, \gamma=u^{r}$, the same argument finishes the proof.

From these two lemmas, we infer the result concerning all the valuations with an irrational Puiseux exponent:

Theorem 14 Let $\partial$ be a holomorphic vector field and $\nu$ a valuation centered in $\mathcal{O}$, with an irrational Puiseux exponent. Let $\left(P_{i}\right)_{i \geq 0}$ be the sequence of infinitely near points of $\nu$. The following conditions are equivalent

a) $\nu$ is a L'Hôpital valuation for $\partial$.

b) $P_{i}$ is a singular point for the strict transform $\partial_{i}$ of $\partial$, for all $i$, and if $P_{k}$ is simple for $\partial_{k}$, then both eigenvalues of its linear part are non-zero and their quotient is not in $(\mathrm{O}) \leq 0$.

A consequence of the results of this section is the following characterization of valuations with an infinite number of Puiseux exponents as those which are never L'Hôpital valuations for any vector field:

Corollary 15 A valuation $\nu$ of $\mathcal{M}$ has an infinite number of Puiseux exponents if and only if there is no vector field for which it is L'Hôpital.

Sufficiency is Corollary 4. The converse is derived from the following lemma, whose prove is done by induction on the number of blowing-ups:

Lemma 16 Let $P$ denote an infinitely near point of $(0,0) \in \mathbb{C}^{2}$ appearing after a finite sequence of blowing-ups $\pi$. Then

(1) If $P$ is a corner, then there exists a singular holomorphic foliation $\omega$ in $\left(\mathbb{C}^{2}, 0\right)$ such that the quotient of eigenvalues of the strict transform $\tilde{\omega}$ at $P$ is not a rational number.

(2) If $P$ is not a corner then, given any (formal or convergent) curve $\gamma$ passing through $P$ and transversal to the exceptional divisor, there exists a singular holomorphic foliation $\omega$ such that: $\gamma$ is a separatrix of $\omega, P$ is a simple singularity of the strict transform $\tilde{\omega}$ of $\omega$, and the quotient of eigenvalues of $\tilde{\omega}$ at $P$ is not in $(0)<0$. 


\section{Rank 1 Valuations and Transcendence of Separatrices}

As valuations of $\mathcal{M}$ of rank one and rational rank one are exactly the divisorial ones, those of contact with a non-convergent curve and those having an infinite number of Puiseux pairs, we have the following corollary, concerning the algebraic independence of solutions of differential equations:

Corollary 17 Keep the notation of the previous sections.

a) There is a valuation $\nu$ of $\mathcal{M}$, of rank 1 and rational rank 1 which is a L'Hopital valuation for a vector field $\partial$ if and only if the foliation defined by $\partial$ is either dicritical or admits a separatrix $\hat{f} \in \hat{\mathcal{O}}$ which is transcendental over $\mathcal{O}$ : that is, $(\hat{f}) \cap \mathcal{O}=(0)$.

b) If $\partial$ is non-dicritical, then there is a bijection between rank 1 and rational rank 1 L'Hôpital valuations and transcendental separatrices: to such a separatrix corresponds its associated contact valuation.

More specifically, for vector fields with polynomial coefficients, by imitating the same arguments as in Theorem 8 and Corollary 17, one can prove:

Corollary 18 Let $\partial=a(x, y) \partial / \partial x+b(x, y) \partial / \partial y$ be a polynomial vector field. Then

a) There is a valuation $\nu$ of $\mathbb{C}(x, y)$, of rank 1 and rational rank 1 which is a L'Hôpital valuation for $\partial$ if and only if the foliation defined by $\partial$ is either dicritical or admits $a$ separatrix $\hat{f} \in \mathbb{C}[[x, y]]$ which is algebraically transcendental over $\mathbb{C}(x, y)$.

b) If $\partial$ is non-dicritical, there is a bijection between rank 1 and rational rank 1 L'Hôpital valuations for $\partial$ and algebraically transcendental separatrices.

c) Moreover, if $\partial$ is regular, the (unique analytic) solution of $\partial$ passing through $(0,0)$ is transcendental over $\mathbb{C}(x, y)$ if and only if there is a L'Hôpital valuation for $\partial$ in $\mathbb{C}(x, y)$ with rank 1 and rational rank 1 .

\section{The "Missing" Separatrices}

In the previous sections, we have characterized some of the separatrices of a germ of analytic vector field in terms of their associated valuations: those having generic or infinite character. The remaining cases include the solution of regular analytic vector fields-which have always rational character. Recall that in the proof of Theorem 10, in order to show that separatrices passing through singularities with rational quotient of eigenvalues did not give rise to L'Hôpital valuations, we chose the functions:

$$
a=u^{m} v^{n+1}, \quad \text { and } \quad b=u^{m} v^{n}
$$

$(u=0)$ being the equation of the separatrix. In fact, any counterexample to L'Hôpital's property must be of that kind: it has to include as a factor, the equation of the separatrix under study. This led us to give the following:

Definition 7 Keep the notation of the previous sections. We say that a valuation $\nu: \mathcal{M}^{\star} \rightarrow \Gamma$ is a weakly L'Hôpital valuation for a vector field $\partial$ if

$$
\nu\left(\frac{a}{b}-\frac{\partial a}{\partial b}\right)>0
$$


for any $a, b \in \mathcal{M}$ with $\nu(a) \geq \nu(b)>0$ and such that if $\nu(c)>0$ then there is $n \in \mathbb{N}$ with $n \nu(c)>\nu(a)$.

This property can be expressed using the notion of isolated subgroups:

Definition Let $\Gamma$ be an ordered group. An isolated subgroup of $\Gamma$ is a subgroup (0) $\neq B \subset \Gamma$ which is a segment: this means that, if $a, b \in B$ and $a \leq c \leq b$, then $c \in B$.

Isolated subgroups are ordered by inclusion, and, its number coincides with the rational rank of the valuation, in our context. The first isolated subgroup is the smallest one. One can see that the first isolated subgroup $\Gamma_{0}$ of a group $\Gamma$ is always $\Gamma$ archimedian, which means that given $a \in \Gamma_{0}$ and $\gamma \in \Gamma$ with $0<\gamma<a$, there is $n \in \mathbb{N}$ such that $n \gamma>a$. In other words, the first isolated subgroup is the largest subgroup "not having infinitely great elements". Thus, Definition 7 becomes:

Proposition A valuation $\nu$ is weakly L'Hôpital for $\partial$ if and only if

$$
\nu\left(\frac{a}{b}-\frac{\partial a}{\partial b}\right)>0,
$$

for any $a, b$ such that $\nu(a), \nu(b)$ are in the first isolated subgroup of $\Gamma$ and with $\nu(a) \geq$ $\nu(b)>0$.

The proof of the following theorem is straightforward

Theorem 19 Let $\nu$ be a valuation centered in $\mathcal{O}$ associated to a curve $f \in \mathcal{O}$ and let $\partial$ be a (germ of) vector field at $\left(\mathbb{C}^{2}, 0\right)$. Then the following conditions are equivalent:

(1) $(f=0)$ is a separatrix of $\partial$.

(2) $\nu$ is a weakly L'Hôpital valuation for $\partial$.

From Theorem 19 we infer the "valuative Cauchy Theorem":

Theorem 20 Let $\partial$ be a regular (germ of) vector field at $\left(\mathbb{C}^{2}, 0\right)$. There is one and only one irreducible (principal) ideal $(f) \in \mathcal{O}$ such that its associated valuation $\nu_{f}$ is a L'Hôpital valuation for $\partial$.

\section{References}

[1] C. Camacho and P. Sad, Invariant varieties through singularities of holomorphic vector fields. Ann. of Math. 115(1982), 579-595.

[2] P. Fortuny, L'Hôpital. Ph.D. thesis, Universidad de Valladolid, 1999.

[3] E. R. Kolchin, Rational approximation to solutions of algebraic differential equations. Proc. Amer. Math. Soc. 10(1959), 238-244.

[4] S. D. Morrison, Continuous derivations. J. Algebra 110(1987), 468-479.

[5] M. Rosenlicht, On the explicit solvability of certain transcendental equations. Inst. Hautes Études Sci. Publ. Math. 36(1969), 15-22.

[6] _ An analogue of L'Hospital's rule. Proc. Amer. Math. Soc. 37(1973), 369-373.

[7] Differential valuations. Pacific J. Math. 86(1980), 301-309.

[8] A. Seidenberg, Reduction of singularities of the differential equation ady $=b d x$. Amer. J. Math. 90(1968), 248-269. 
[9] Derivations and valuation rings. In: Contributions to algebra (eds. Bass, Cassidy, Kovacic), Academic Press, New York, 1977, 343-347.

[10] M. F. Singer, Linear differential equations in function fields. Proc. Amer. Math. Soc. 54(1976), 69-72.

[11] M. Spivakovsky, Valuations in function fields of surfaces. Amer. J. Math. (1) 112(1990), 107-156.

[12] M. Vaquié, Valuations. In: Resolution of singularities (eds. H. Hauser et al.), Progr. Math. 181, Birkhäuser, 2000, 541-590.

[13] O. Zariski, The reduction of singularities of an algebraic surface. Ann. of Math. 40(1939), 639-689.

\author{
School of Mathematical Sciences \\ Queen Mary College \\ University of London \\ Mile End Road \\ London E1 4NS \\ $U K$ \\ email: pf@maths.qmul.ac.uk
}

